\title{
Tumor Odontogénico Adenomatoide: Reporte de dos casos de localización mandibular
}

\begin{abstract}
Resumen
El Tumor Odontogénico Adenomatoide (TOA) es una neoplasia benigna que constituye entre el 3 al 7\% de todos los tumores odontogénicos. Se asocian generalmente a dientes incluídos, afectando mayoritariamente al maxilar superior en el sector anterior, caracterizándose por su crecimiento lento, indoloro y asintomático. Se
\end{abstract}

Oriana-Valenzuela- Rivera ${ }^{1}$

María Angélica-Vila- Valenzuela²

Relato de caso

\section{Tumor Odontogênico Adenomatóide: Relato de dois casos de localização mandibular}

\section{Resumo}

O Tumor Odontogênico Adenomatóide (TOA) é uma neoplasia benigna que atinge de 3 a $7 \%$ de todos os tumores odontogênicos. Associam-se geralmente a dentes inclusos, afetando majoritariamente o maxilar superior na região anterior, caracterizando-se por crescimento lento, indolor e assintomático. São apresentados dois ca- presentan dos casos de pacientes jóvenes de 13 y 11 años de sexo masculino y femenino respectivamente con Tumor Odontogénico Adenomatoide, ambos ubicados en el sector posterior de la mandíbula y asociados a dientes incluídos. El estudio inmunohistoquímico de uno de los dos casos presentados demostró positividad para Ki-67 de un 12-20\% patrón nuclear. 
Case report

\section{Adenomatoid Odontogenic Tumor: Report of two cases of mandibular location}

\begin{abstract}
Adenomatoid Odontogenic Tumor (AOT) is a neoplasia which is between 3 to $7 \%$ of all odontogenic tumors. Teeth are usually associated included, affecting mostly the upper jaw in the anterior, characterized by slow growth, painless and asymptomatic. We present two cases of young patients of 13 and 11 years male and female respectively Adenomatoid Odontogenic Tumor, both located in the posterior region of the mandible and associated teeth included.

Immunohistochemical study of one of the two cases presented showed positivity for Ki-67 of a $12-20 \%$ nuclear pattern.
\end{abstract}

Keywords: Adenomatoid odontogenic tumor, dentigerous cyst.

\section{Introducción}

El Tumor Odontogénico Adenomatoide (T.O.A.) es una neoplasia epitelial benigna de origen odontogénico, relativamente poco frecuente, representa alrededor del 3 al 7\% (1)(33) de todos los tumores odontogénicos, su ubicación mas frecuente es en relación a la corona de caninos superiores incluídos(3)(5)(31). Aproximadamente el $75 \%$ de los casos se presentan en la región anterior de los maxilares y un $65 \%$ de los casos en el maxilar superior.(33) Se presenta con mayor frecuencia en mujeres que varones, en una relación 2:1, más de un tercio son diagnosticados en la segunda década de la vida, manifes- tándose como aumento de volumen indoloro de crecimiento lento $(2)(4)(3)(1)(30)(8)$, no exceden a $3 \mathrm{~mm}$. de diámetro (1)(2(33).

Se reportan casos de tumores odontogénicos en niños y adolescentes en Nigeria, del total de 78 casos estudiados un $9 \%$ correspondió a TOA(35), un estudio retrospectivo de 349 de tumores odontogénicos en México, dio como resultado de un 7,1\% del total de los casos (36).

En el pasado esta lesión fué descrita como una variedad del ameloblastoma, siendo denominada adenoameloblastoma, posteriormente se reconoce como una entidad patológica diferente de acuerdo a su naturaleza, comportamiento e histología(5)(30)(3). Algunos autores postulan que de acuerdo al patrón de crecimiento y lo circunscrita de la lesión puede ser considerada o clasificada como hamartoma más que una verdadera neoplasia (21)(31).

Las células tumorales del T.O.A. derivan del epitelio reducido del órgano del esmalte de la fase postsecretora del desarrollo del órgano del esmalte (6)(30), del estrato intermedio, otros investigadores sugieren que la lesión deriva de remanentes de la lámina dental (1) (30).

Existen tres subvariedades clínicas las cuales comparten la misma histología: la forma folicular que representa el 73\% de los T.O.A. de localización central, asociado a diente impactado; la forma extrafolicular de localización central, que no se asocia a estructura dentaria representa el $24 \%$ y la forma periférica que afecta la mucosa 
gingival que representa al 3\% de los T.O.A. (2) (7)(13).

Desde el punto de vista histopatológico el T.O.A. presenta una cápsula externa de tejido conjuntivo fibroso que rodea una formación nodular de células epiteliales , esta característica le confiere un carácter no invasivo.(34). El resto del tejido puede ser sólido o contener áreas quísticas focales, los nódulos están constituídos por células epiteliales fusiformes las que se disponen en forma arremolinada, en roseta, con mínima cantidad de tejido conjuntivo del estroma(24,2). Dispersas en la lesión se encuentran células cilíndricas que conforman estructuras pseudoductales, entre las células epiteliales es frecuente encontrar material eosinófilo amorfo (4)(7)(6)(2), que algunos autores denominan "gotas eosinófilas" (2), ocasionalmente pueden aparecer calcificaciones esféricas interpretadas como formación de esmalte abortivo (2)(24)(31).

Estudios inmunohistoquímicos de Ki-67 indican baja actividad proliferativa de la lesión lo que explicaría la no recurrencia de la lesión (2)(29).

Radiográficamente los T.O.A. foliculares se presentan como lesiones radiolúcidas uniloculares circunscritas que rodean la corona dentaria de

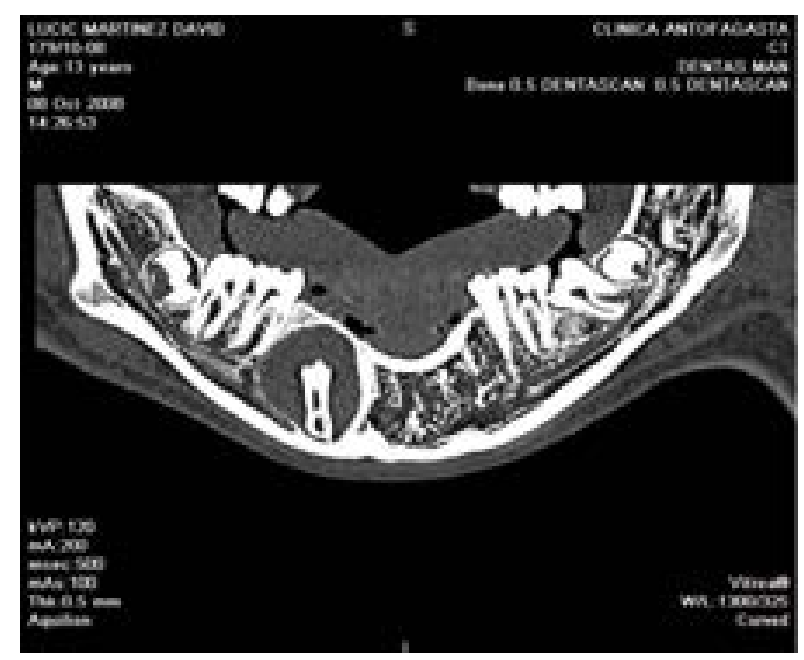

Figura 1 a y $b$. un diente no erupcionado, similar al quiste dentígero(3)(5)(28), la lesión puede expandir tablas, se extiende apicalmente a lo largo de la raíz bajo la unión cemento - esmalte, hallazgo que permite realizar el diagnóstico diferencial con Quiste dentígero (1)(4).

Se presentan dos casos clínicos de Tumor Odontogénico Adenomatoide de pacientes atendidos en el Servicio Dental del Hospital Regional de Antofagasta; ambos de localización poco frecuente, de variedad folicular que comprometen mandíbula y están asociados a dientes 4.4 y 3.7 intraóseos respectivamente.

\section{Caso 1}

Paciente varón de trece años de edad, sin antecedentes mórbidos de importancia, derivado por cirujano-maxilofacial para estudio imagenológico, con diagnóstico de aumento de volumen vestibular en relación a diente 4.4, persistencia de diente 8.4. Asintomático.

Al exámen intraoral se observa aumento de volumen a expensas de la tabla vestibular del cuerpo mandibular derecho, en relación a diente 4.4; la mucosa se presenta de aspecto normal.

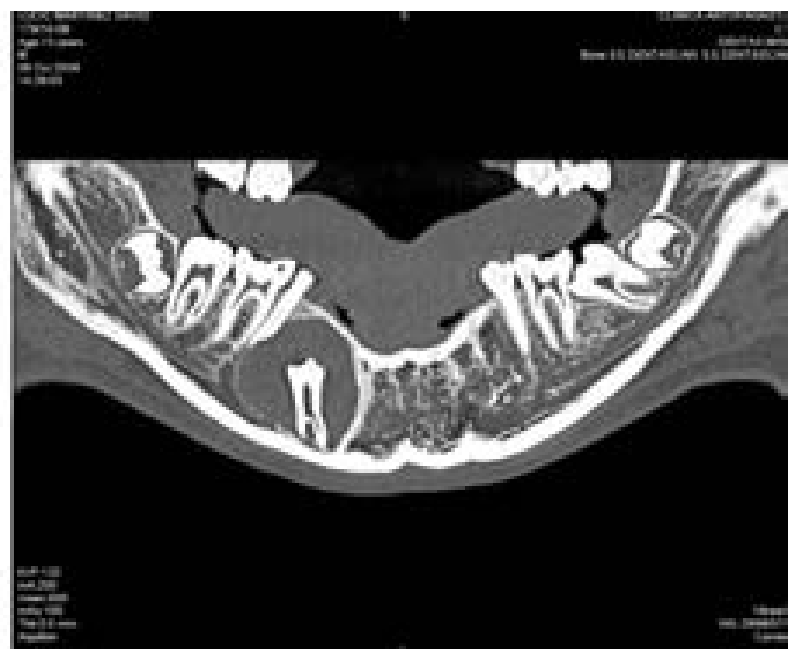


Imagenología: A la tomografía computarizada se observa lesión expansiva, hipodensa, redondeada corticalizada, asociada a diente 4.4, biradiculado e incluído, que se encuentra en posición oblicua con la corona hacia distal, la pieza dentaria se encuentra desplazado hacia basilar, la cortical distal se presenta adelgazada. Fig. 1a, 1b. Rodeando la corona se observan pequeñas calcificaciones periféricas. Fig. 2. La lesión rodea toda la pieza dentaria, expande tablas óseas, observándose exteriorización de la lesión hacia vestibular. Fig. 3.

Las raíz del diente 4.5 se encuentra ligeramente desplazada a distal.

El tamaño de la lesión es de $26.1 \mathrm{~mm}$. en sentido mesio - distal y de $24.3 \mathrm{~mm}$. en sentido vestíbu-

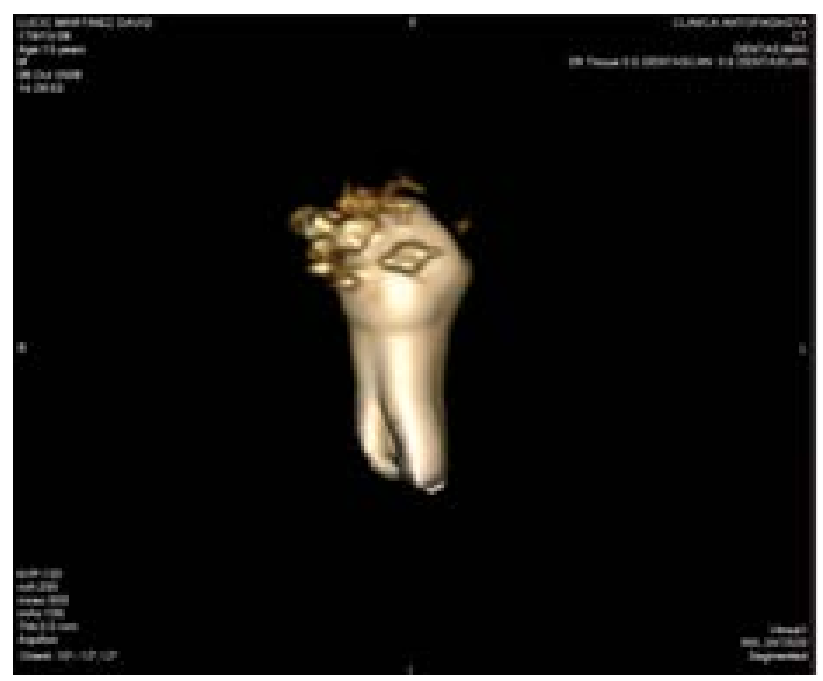

Figura 2.

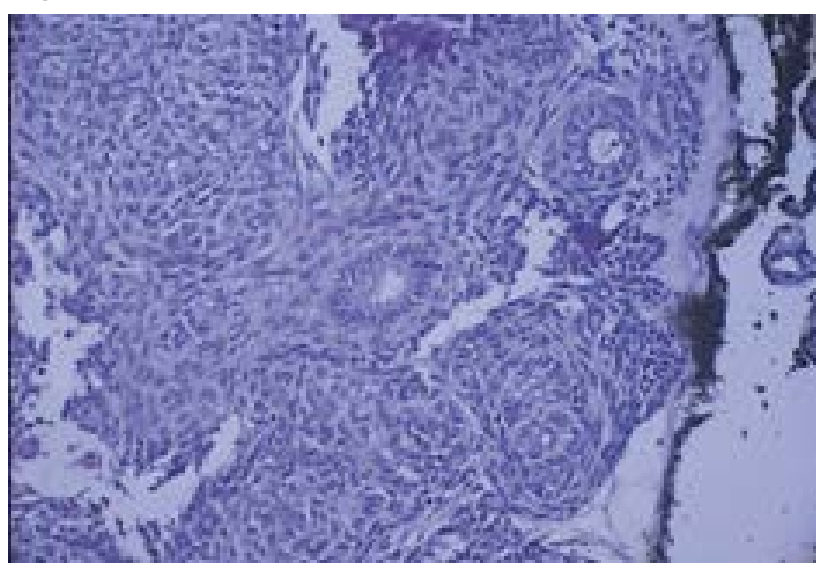

Figura $4 a$ y $b$. lo - lingual y se encuentra en relación a conducto dentario.

Se miden densidades alcanzando valores de 100.8, 96.3 y 77.3 U.H. respectivamente.

El diente 3.7 se encuentra semi - incluido y en mesioversion.

Histopatología: La muestra comprende tejido compuesto por epitelio odontogénico que se dispone en acúmulos sólidos, arremolinados, con estructuras pseudocanaliculares y pequeños quistes o estructuras tubulares. El tejido presenta calcificaciones y acúmulo de material eosinófilo con aspecto de dentina displásica. Fig. 4 a, b.

Diagnóstico Histopatológico: Tumor Odontogénico Adenomatoide.

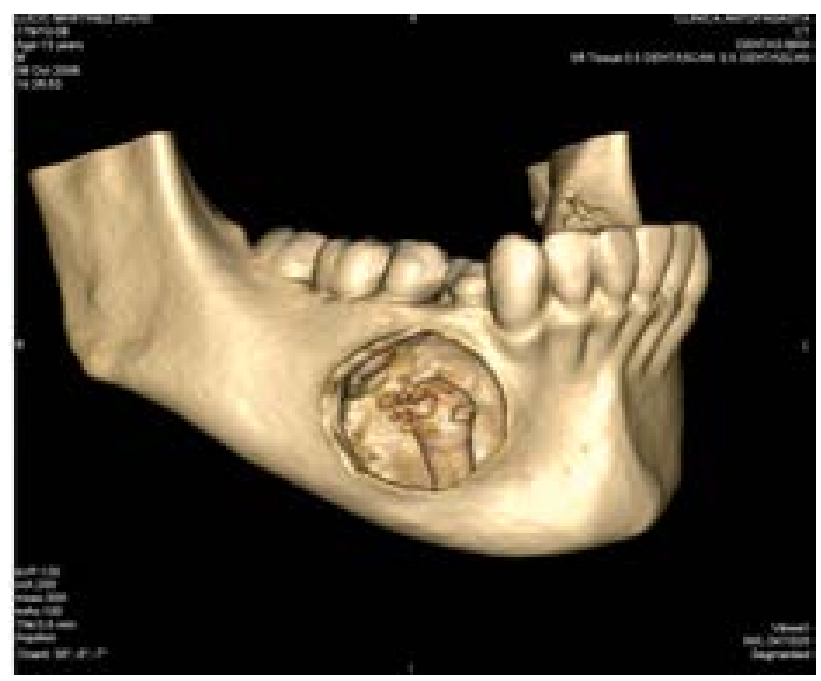

Figura 3.

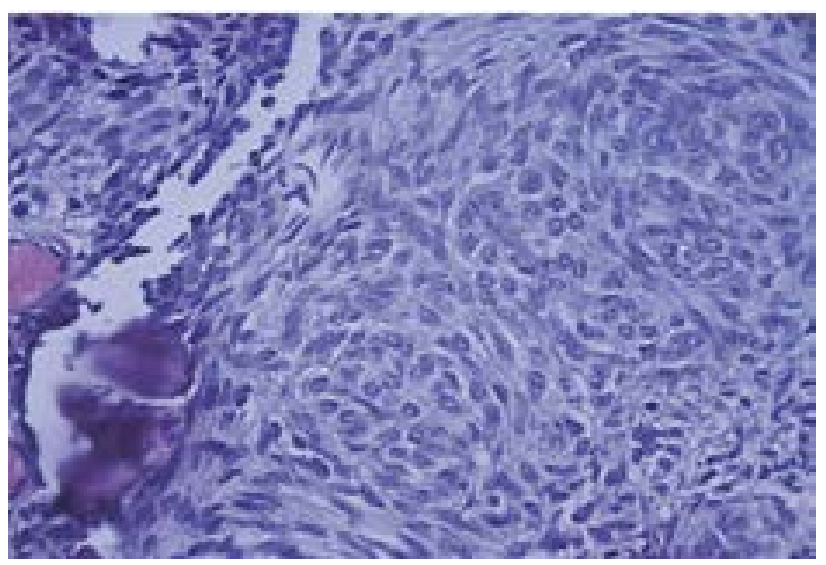




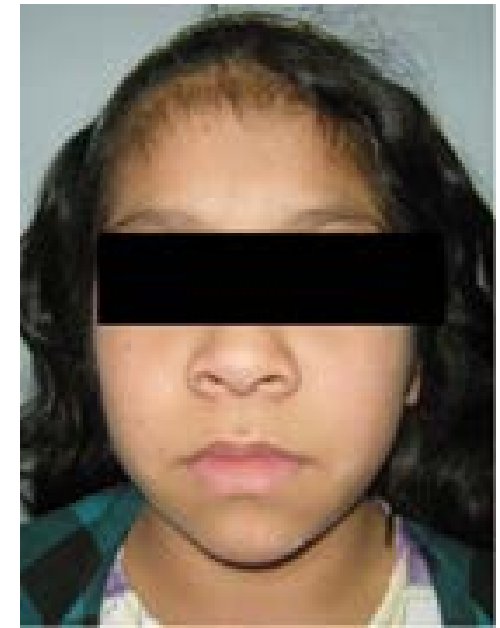

Figura 5.

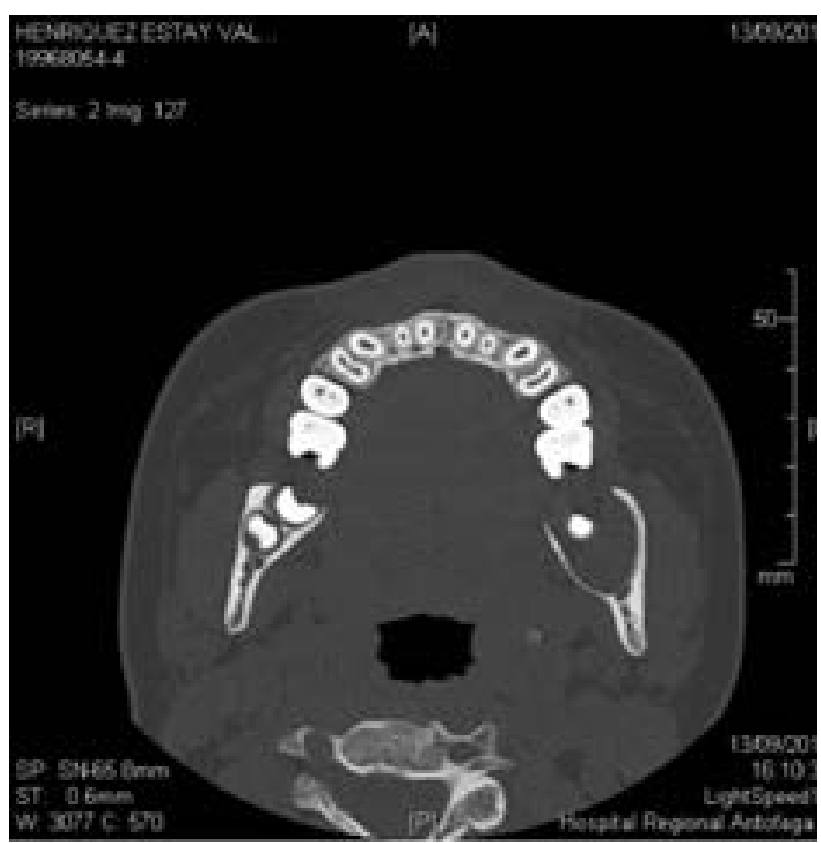

Figura 7 a y $b$.

\section{Caso 2}

El presente caso corresponde a un hallazgo radiográfico en un paciente sexo femenino de 11 años, sin antecedentes mórbidos de importancia, derivada de la Especialidad de Ortodoncia para estudio radiográfico previo a tratamiento de Ortodoncia.

Al examen clínico extraoral se observa discreta asimetría facial con ligero aumento de volumen región facial izquierda. Fig. 5.

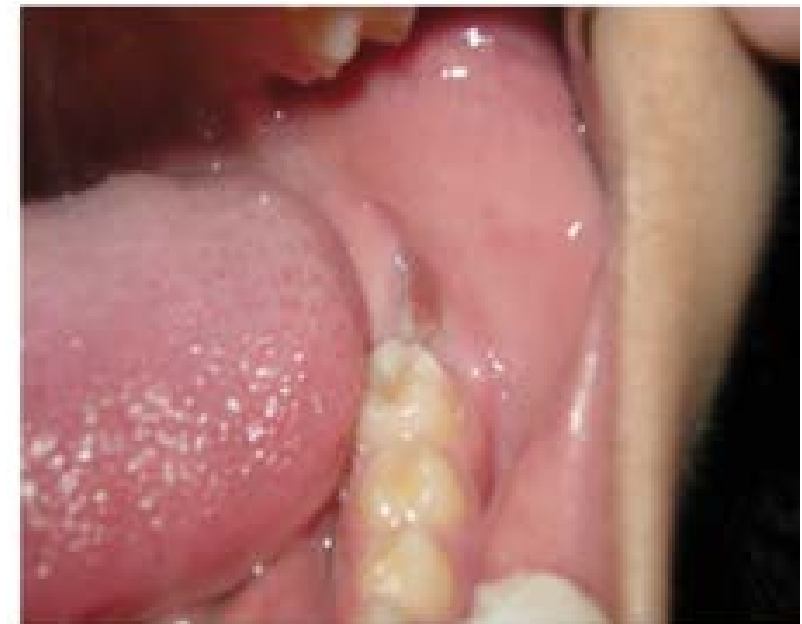

\section{Figura 6.}

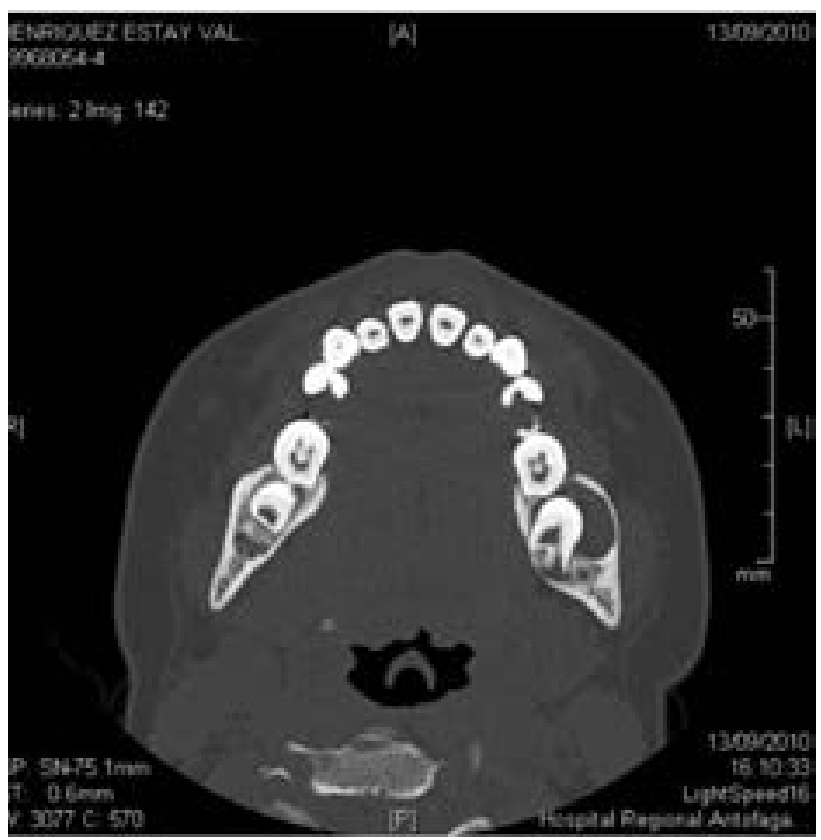

$\mathrm{Al}$ examen clínico intraoral la mucosa se observa de características normales, en el fondo de vestíbulo distal a diente 3.6 se observa lesión ulcerada. Fig. 6.

A la palpación en la zona retromolar se observa abombamiento de las tablas vestibular y lingual.

Imagenología: En la tomografía computarizada, distal a diente 3.7, el que se encuentra intraóseo con desarrollo de 2/3 radiculares, desplazado hacia lingual. Fig. 7 a, b. Se observa una lesión 

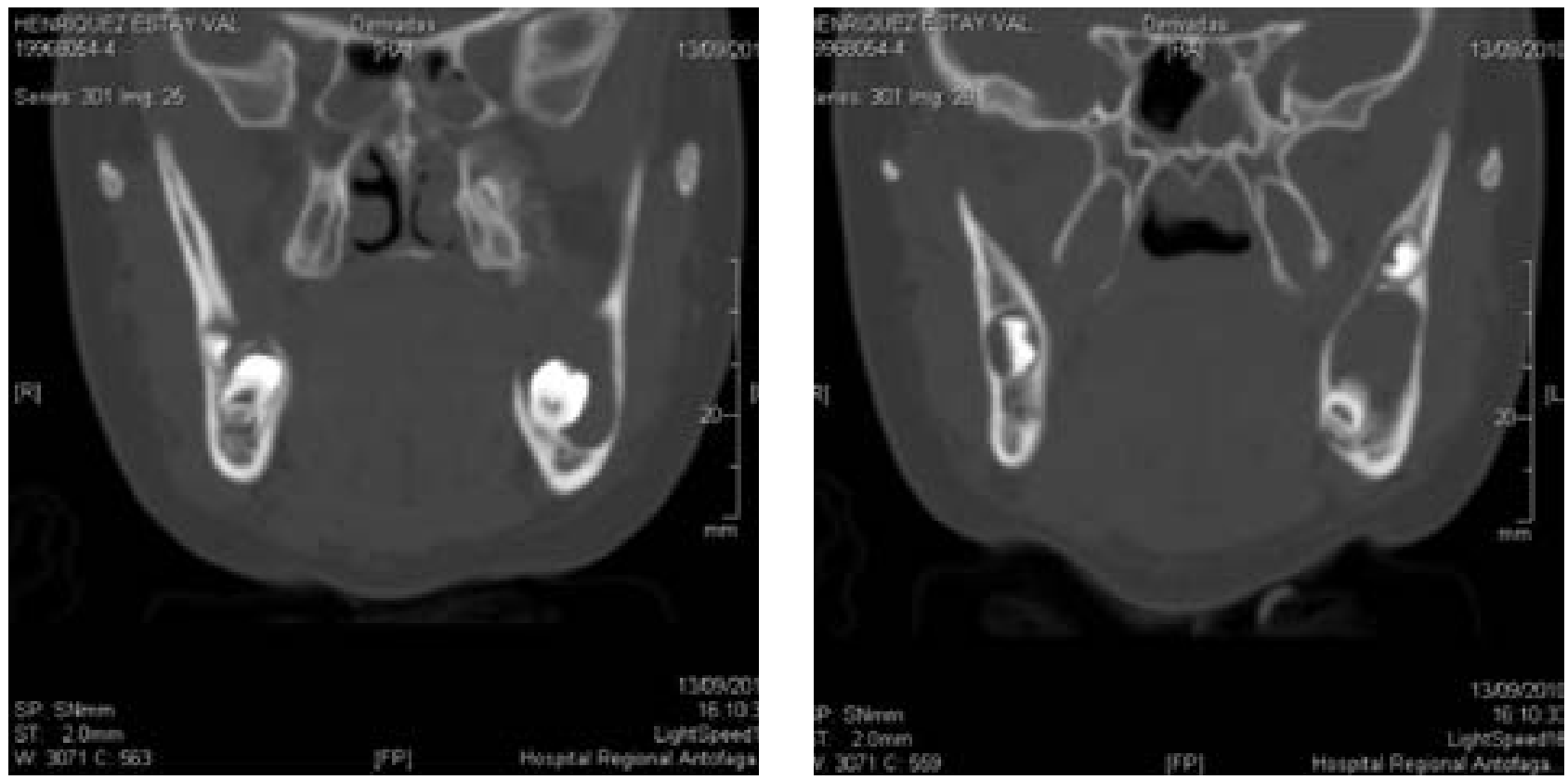

Figura $8 a$ y $b$.

radiolúcida unilobulada con una extensión de $16.76 \mathrm{~mm}$. en sentido vestíbulo-lingual y de $30.07 \mathrm{~mm}$. en sentido mesio-distal. La lesión se extiende desde la zona coronoradicular mesial de diente 3.7 hacia distal, desplazando a diente 3.8 hacia la rama mandibular, el diente 3.8 se encuentra con $2 / 3$ coronarios calcificados, la lesión se observa adelgazada hacia lingual y en íntima relación con el conducto alveolar inferior. Fig. 8 $a, b$.

Histopatología: formación quística revestida parcialmente por epitelio escamoso y con estroma edematoso fibrovascular y neoplasia sólida de estirpe epitelial con células fusadas de distribución nodular con escaso componente estromal.

\section{Estudio inmunohistoquímico:}

- Citoqueratina (AE1 - AE3) POSITIVO difuso en células neoplásicas, patrón citoplasmático. Fig. 9.
- S-100 POSITIVO en una proporción de células neoplásicas, patrón nuclear y citoplasmático. Fig. 10.

- Ki67 POSITIVO alrededor del 12 - 20\% de células neoplásicas patrón nuclear. Fig. 11.

Diagnóstico Histopatológico: Tumor odontogénico adenomatoide con componente predominantemente fusocelular.

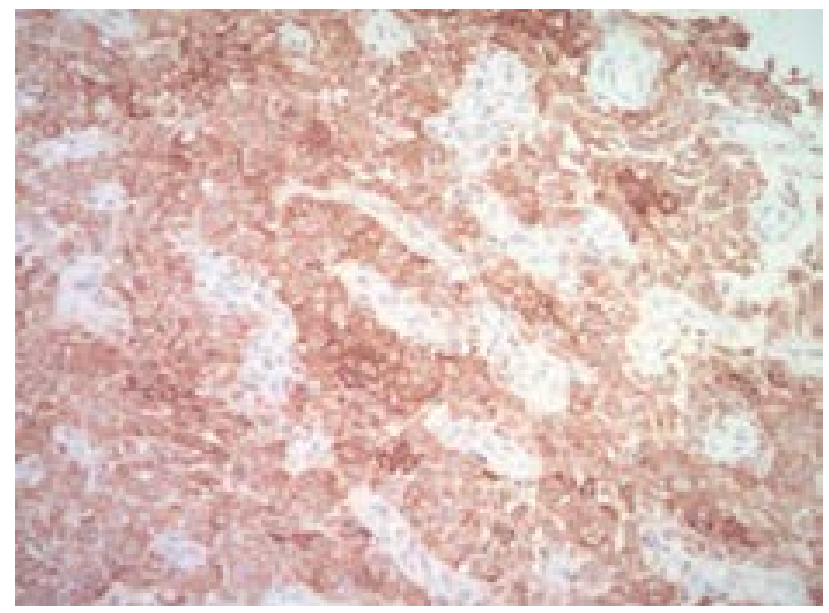

Figura 9. Citoqueratina. 


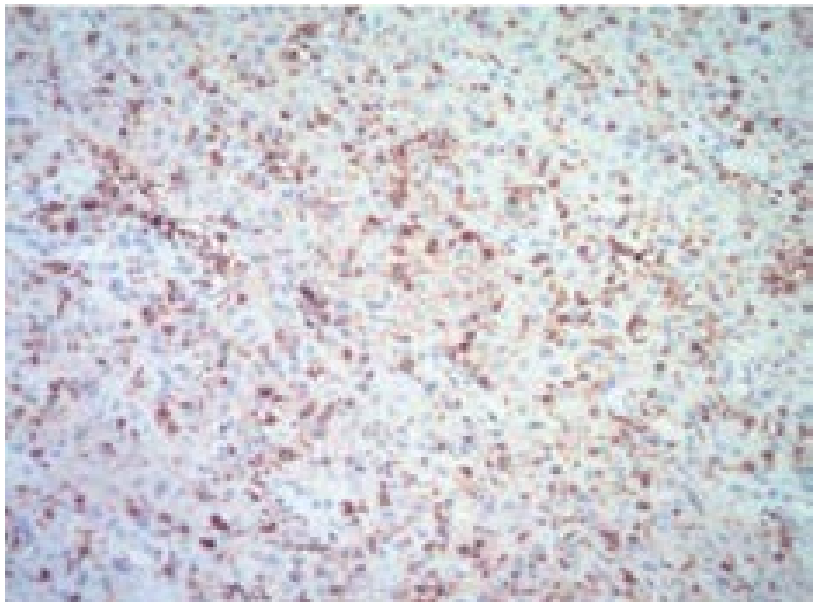

Figura 10. S-100.

\section{Discusión}

El TOA antes de ser clasificado como una neoplasia verdadera, algunos autores lo consideraron una lesión hamartomatosa $(21)(31) \sin$ embargo en la nueva clasificación de Tumores Odontogénicos del año 2005 el TOA es definido como una neoplasia benigna de crecimiento lento y progresivo (9).

Se postula que el origen del TOA es a partir del epitelio odontogénico del órgano del esmalte (22) o de remanentes de la lámina dental (17), la calificación de adenomatoide está dada por el patrón histológico con presencia de estructuras pseudoductales $(30,1,31,10,11,12)$.

Los casos presentados corresponden a la variante central intraósea folicular de acuerdo a la reclasificación del TOA de Philipsen et al. realizada en el año 1991 (15) El TOA presenta un crecimiento lento, generalmente asintomático, característica observada en los dos casos expuestos, éstos difieren a lo encontrado en la literatura respecto a la localización ya que ambos comprometían a la mandíbula; el momento del diagnóstico fue en la segunda década de vida. Estudios indican que mayoritariamente (65\%)

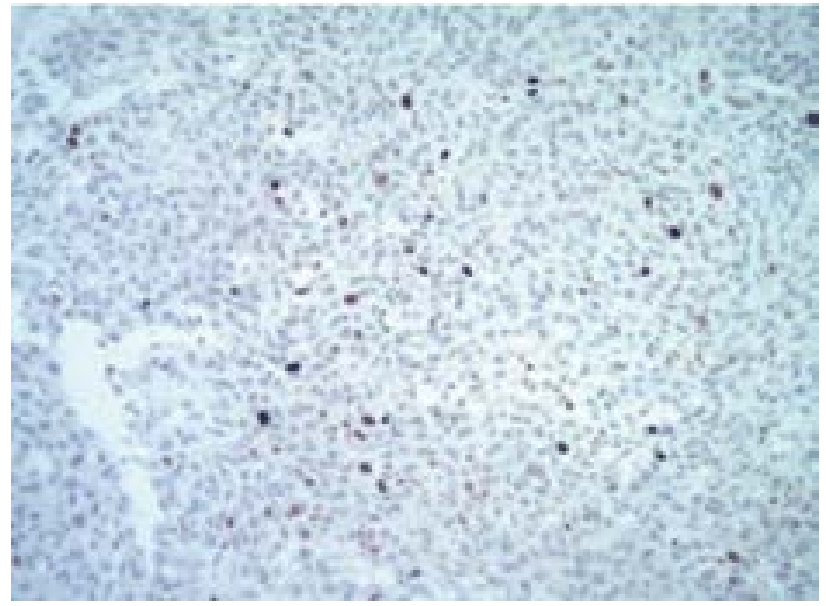

Figura 11. Ki67.

los TOA son diagnosticados en la segunda década y que comprometen el maxilar, en una relación de 2:1 respecto a la mandíbula. $(33,26)$

Los TOA aquí presentados se encuentran dentro del tamaño promedio descrito en la literaura de 1,5 a $3 \mathrm{~cm}$ de diámetro (21) (27). La manifestación a nivel mandibular es poco frecuente, sin embargo se han descrito otros casos en localización anteroinferior (19)(20)(25). El aumento de volumen mandibular a expensas de la tabla vestibular, la persistencia del diente 8.4 asociado a la alteración de la cronología de erupción del diente 4.4 en el caso 1 orientan a la presencia de un proceso patológico a evaluar, el que se evidencia al examen imagenológico. El segundo caso presentado correspondió a un hallazgo radiográfico en un examen imagenológico de rutina previo a tratamiento de Ortodoncia, que luego al examen clínico intraoral se pudo observar una lesión de la mucosa de aspecto ulcerado en relación directa con la lesión ósea, que compromete al diente 3.7 el que se encuentra retenido y que además desplazaba a diente 3.8 hacia la rama mandibular, característica de desplazamiento dentario similar a otros casos reportados en la literatura (13). En ambos casos se observan 
piezas dentarias retenidas imagen frecuente de esta lesiòn $(31,1,30,32)(13)$ la mayoría de los casos que se reportan están asociados a caninos maxilares en un porcentaje entre un 40 a un $70 \%$ $(14,15)$, los dos casos presentados estàn asociados a premolar y molar mandibular respectivamente, ubicación menos frecuente de esta patología, como casos reportados por Layton (16) y Philipsen 1992 (17).

En uno de los casos presentados se observó la presencia de calcificaciones orbitando la corona dentaria, patrón que permite realizar diagnóstico diferencial con quiste dentígero, además de la extensión de la lesión involucrando la raíz en el caso del TOA. $(18,5,6,23)$

En ambos casos el tratamiento realizado fue la enucleación de la lesión, se ha realizado seguimiento de los pacientes, no presentando recidiva a la fecha, lo cual concuerda con Giansanti et al. (14) quien reporta seguimientos de casos durante 10 años sin observar recurrencia. La recidiva de estas lesiones estaría asociada a la baja positiividad a Ki-67 en estudios de inmunohistoquímica, se podría traducir como baja actividad proliferativa del tumor (2). En el caso 2 la positividad a Ki-67 resultó de un 12-20\% patrón nuclear, valor que supera lo reportado en la literatura de un $2-3 \%$ de positividad (2) resultado que debemos considerar para el seguimiento estricto de este caso en particular, ya que aunque poco frecuente se ha descrito recurrencia (4).

El TOA constituye un tumor odontogénico que se presenta con poca frecuencia, los casos aquí presentados corresponden a la variante intraósea central folicular asociados a dientes retenidos en región premolar y molar mandibular. Los análisis histopatológicos confirmaron el diagnóstico de TOA y a la fecha mediante controles imagenólogicos post-quirúrgicos no se ha demostrado recidiva.

\section{Referencias}

1. Neville B, Damm D, Allen C, Bouquot J. Oral \& Maxillofacial Pathology. W.B. Saunders Company 2009;713-715.

2. Neville B, Damm D, White D. Atlas Colorido de Patología Oral Clínica. Distúrbios do osso. Segunda Edición. $2001 ; 406-407$.

3. Som P, Curtin H. Radiología de cabeza y cuello. Mosby $4^{a}$ Edición 2004;1:952-53.

4. Freitas A, Rosa J, Farías e Souza I. Radiología Odontológica. Artes Médicas Latinoamericana. $1^{\text {a }}$ Edición $2002 ; 477$.

5. Soames JV, Southam JC. Oral Pathology. Odontomes and Odontogenic tumours. Oxford 4ª Edición 2005-2006;232-233.

6. Vera FJ, Artes MJ, Vera B \& Bonet J. Tumor odontogénico adenomatoide folicular: Estudio inmunohistoquímico. Med. Oral patol. Oral cir. Bucal 2006;11:4.

7. Chuan-Xiang Z, Gan G. Adenomatoide tumor odontogénico: a propósito de un caso poco frecuente, con recurrencia. J Med Oral Pathol 2007;36(7):440-3.

8. Sciubba JJ, Fantasia JE, Kahn L. Atlas of Tumor Pathology. Tumors and Cysts of the Jaw. Benign Odontogenic Tumors 2001; 90-95.

9. Philipsen HP, Nikai H. Pathology and Genetics of Head and Neck Tumours. Chapter 6. Odontogenic Tumours 2005;304-305.

10. Adebayo ET, Ajike SO, Adekeye EO. Odontogenic tumours in children and adolescents: a study of 78 Nigerian cases. J Craniomaxillofac Surg 2002;30(5):267-72.

11. Mosqueda-Taylor A, Ledsma Montes C, Cabllero-Sandoval S, Portilla-Robertson J, Ruiz-Godoy Rivera LM, MenesesGarcía A. Odontogenic tumors in Mexico: a collaborative retrospective study of 349 cases. Oral Surg Oral Med Oral Pathol Oral Radiol Endod. 1977;84(6):672-5.

12. Philipsen HP, Reichart PA. Adenomatoid odontogenic tumor: facts and figures. Oral Oncol 1998;35:125-31. 
13. Saap JP, Eversole LR, Wysocki GP. Patología Oral y Maxilofacial Contemporánea. Harcourt Brace. 1998;136-38.

14. Philipsen HP, Reichart PA, Siar CH, Ng KH, Lau SH, Zhang X, Dhanuthai K, Swasdison S, Jainkittivong A, Meer S, Jivan V, Altini M, Hazarey V, Ogawa I, Takata T, Taylor AA, Godoy H, Delgado WA, Carlos-Bregni R, Macías JF, Matsuzaka K, Sato D, Vargas PA, Adebayo ET. An updated clinical and epidemiological profile of the adenomatoid odontogenic tumor: a collaborative retrospective study. J. Oral Pathol Med 2007;36:383-93.

15. Velasco, I, Aguilar L. \& Venables C. Adenomatoid odontogenic tumor in maxilla: a report case and review of the literature. Int. J. Odontostomat 2011;5(1):65-69.

16. Boraks,S. Diagnóstico Bucal. Semiología y alteraciones de los huesos maxilares. Artes Médicas Latinoamérica $2004 ; 283$.

17. Escalante M, Rebolledo M. Concepto actual, diagnóstico y tratamiento del tumor odontogénico adenomatoide.Reporte de un caso. Saud Uninorte. Barranquilla (Col.)2012;28(3):432-437.

18. León JE, Mata GM, Fregnani ER, Carlos-Bregni R, de Almeida Op, Mosqueda-Taylor A, Vargas PA. Clinicopathological and Inmunohistochemical study of 39 cases of Adenomatoid Odontogenic Tumor: a multicentric study. Oral Oncol. 2005;41(8): 835-42.

19. Ortiz E, Liceaga C, Mosqueda A, Liceaga R. Tumor odontogénico adenomatoide: presentación de un caso de tamaño inusual y revisión de literatura. Rev Hosp Jua Mex 2006;73(2):60-63.

20. Barnes L, Eveson J.W., Reichart P \& Sidransky D. World Health Organization classification of tumors.Pathology and genetics of head and neck tumors. Lyon, IARC, 2005.

21. Schlosnagle DC, Someren A. The ultrastructure of the adenomatoid odontogenic tumor. Oral Surg Oral Med Oral Pathol 1981;52:154-60.

22. Philipsen HP, Samman N, Ormiston IW, Wu PC, and Reichart PA. Variants of the adenomatoid odontogenic tumor with a note on tumor origin. J Oral Pathol Med 1992;21:348-52.

23. Bascones A, Llanes F. Medicina Bucal. Segunda Ediciòn. Tomo II Ediciones Avances Medico-Dentales, S.L. 1991;527-528.

24. Goaz \& White. Oral Radiology. Principles and Interpretation. Tercera Edición 1995;87-95.

25. Langlais R, Langland O, Nortjé Ch. Diagnostic Imaging of the Jaws. Williams \& Wilkins. 1995;312-315.

26. Philipsen HP, Reichart PA, Zhang KH, Nikai H, Yuq X. Adenomaoid Odontogenic Tumor: Biologic profile based on 499 cases. J Oral Pathol Med 1991;20(4):149-158.

27. Briones D, Basili A, Castellón L, Montero S. Tumor Odontogénico Adenomatoide: Reporte de Caso y Revisión Bibliográfica. Revista Dental de Chile 2005;96(2):14-16.

28. Lee JK, Lee KB, Hwang BN. Adenomatoid Odontogenic Tumor: A Case Report. J Oral Maxillofac Surg 2000;58:1161-1164.

29. Sanchez E, Vila D, Garmendia A, Serra A, Torres A. Tumor odontógeno adenomatoide en region mandibular. Revista Cubana de Estomatología 2010; 47(4):447-454.

30. Montilla G., Rivera H. Tumor odontogénico adenomatoide. Reporte de un caso en Mandíbula-Revisión bibliográfica. Acta Odontológica Venezolana 1997;35:3.

31. de Almeida R, Carneiro B, Costa L, Silveira de Castro C. Tumor odontogénico adenomatoide en mandíbula. Revista Cubana de Estomatología 2011;48(2):172-180.

32. Regezi JA, Sciubba JJ, Jordan R. Oral Pathology. Clinical Pathologic Correlations. Chapter 11. Odontogenic Tumors 2003; 276-277.

33. Giansanti JS, Somersen A, Waldron CA. Odontogenic adenomatoid tumor (adenoameloblastoma). Oral Surg Oral Med Oral Pathol 1970;30:69.

34. Layton SA . Adenomatoid odontogenic Tumor: report of an unusual lesion in the posterior maxilla. Dentomaxillofac Radiol 1992;21(1): 50-52.

35. Dare, A., Yamaguchi, A., Yoshiki, S. \& Okano, T. Limitation of panoramic radiography in diagnosing adenomatoid odontogenic tumors. Oral Surg. Oral ME. Oral Pathol 1994;77(6):662-8.

36. Alfaro L, Martínez B. Atlas de Patología de los maxilares. 1ª Edición (Madrid); Ripano S.A. 2011;150-54.

Revisión y traducción de portugués: Fabián Calixto Fraiz

Recibido: 26-06-13

Aceptado: 03-07-13

Correspondencia: draoriana@gmail.com 


\section{INSTRUCCIÓN PARA LOS AUTORES / INSTRUCTIONS FOR THE AUTHORS}

\section{Información general}

La Revista de Odontopediatría Latinoamericana es la publicación oficial de la Asociación Latinoamericana de Odontopediatría (ALOP) siendo dirigida a profesionales y estudiantes de odontología y áreas afines que estén interesados en la atención a la salud de niños y adolescentes. Ella es publicada dos veces por año en forma ininterrumpida, su objetivo es la divulgación de investigación y conocimiento en odontopediatría y áreas afines. El Comité de Redacción y el Consejo Editorial sigue los requisitos establecidos por el Comité Internacional de Editores de Revistas Biomédicas, publicado en 1997 (Directivas de Vancouver) (http: / / www.icmje.org/).

\section{Instrucción para los autores}

La revista acepta trabajos en las siguientes modalidades: artículos de inbestigaxión, artículos de revisión, relatos de caso, comunicaciones previas, cartas al editor. Serán considerados para publicación solamente artículos originales. Los trabajos originales deben ser enviados al Editor electrónicamente, solicitando apreciación para publicación e informando en carta de remisión que el material no fue publicado anteriormente y no está siendo considerado para publicación en otra revista, cualquier sea en el formato impreso o electrónico. La decisión de aceptación para publicación es de responsabilidad de los Editores y se basa en las recomendaciones del cuerpo editorial y/o revisores "ad hoc".

Los principios éticos de investigación definidos por la Declaración de Helsinki deberán ser respetados. Los autores deben describir en la sección de Material y Métodos la aprobación por los Comités de ética en investigación de la Institución donde la fue realizada.

\section{Proceso de revisión y evaluación de manuscritos}

Todos los artículos encaminados serán sometidos al análisis de por lo menos dos evaluadores.

1. En un primer momento, los trabajos serán evaluados por los editores en cuanto al cumplimiento de las normas editoriales y verificación de adecuación a los objetivos de la revista. En caso de cumplidos los requisitos será atribuido un código que lo identificará en las etapas siguientes. Durante todo el proceso de tramitación de los artículos, tanto evaluadores cuánto autores, no serán identificados por la otra parte.

2. Las obras que atiendan a los requisitos serán encaminadas al Comité de Evaluadores para apreciación en cuanto al mérito, método científico y precisión estadística. Si hubiera divergencia entre los evaluadores, el Editor podrá solicitar una tercera opinión.

3. El evaluador irá a emitir su parecer indicando si el manuscrito fue: a) aceptado, b) aceptaso después de las correcciones sugeridas, c) rechazado para publicación.

4. Los autores cuyas obras necesitan de correcciones deben realizarlas y devolver al editor con una carta aceptando las sugerencias o exponiendo las razones para no acatarlas.

5. El Editor con base en la respuesta de los evaluadores aprobará o rechazará el manuscrito y comunicará su decisión a los autores.

6. Los trabajos aprobados serán revisados y adecuados al formato de la revista por el Editor y Consejo Editorial, la publicación será en consonancia con las prioridades y la disponibilidad de espacio. Una vez aceptado y publicado los derechos de la obra pertenecen a la Revista de Odontopediatría Latinoamericana. Las opiniones y conceptos emitidos, así como el contenido de los textos de las citaciones y referencias bibliográficas son de responsabilidad de los autores, no reflejando necesariamente la opinión del Cuerpo Editorial y de los Editores. 


\section{Tipos de Publicación}

- Editorial: es un texto escrito por el editor o autor invitado, donde se discute una temática de especial importancia para la odontopediatría, incluyendo sus cuestiones institucionales.

- Artículos de investigación: son publicaciones originales concluidas sobre temas de interés de la especialidad. Describe nuevos descubrimientos en el formato de un trabajo que contiene informaciones que permitan la confirmación de sus resultados.

- Artículos de revisión: es una revisión de la literatura actualizada sobre un tema con un análisis crítico y objetiva sobre el estado actual del conocimiento. Compilan el conocimiento disponible sobre un determinado tema, contrastando opiniones de varios autores eincluyendo una profundizada y crítica pesquisa bibliográfica.

- Relato de casos: debe ser un relato sucinto y claro deinterés especial, conteniendo introducción, descripción del caso o serie de casos, discusión y conclusiones. Debe ser acompañada por ilustraciones esenciales.

- Cartas al Editor: son comentarios, observaciones, críticas y sugerencias sobre los artículos publicados o argumentos de interés de los lectores, siempre basado en evidencias científicas referenciadas.

- Comunicaciones previas: son resultados preliminares de trabajos de investigación

\section{Presentación del manuscrito}

La obra debe ser redactada en español y portugués (digitalizados en programas compatibles con "Microsoft Word sea Windons") en fuente Arial 12, espacio doble con márgenes de 2,5 centímetros y página tamaño A4. Las páginas, con la salvedad de la hoja de presentación, deben ser numeradas y estructuradas en la siguiente secuencia.

1. Hoja de presentación, conteniendo:

- Título del trabajo (máximo de 50 caracteres con espacios y solamente la primera palabra en mayúscula);

- Nombre completo de los autores, seguido de su principal titulación y filiación institucional y correo electrónico (se existen más de 6 autores debe ser presentado justificación);

- Dirección completa (incluyendo teléfono) del autor principal

2. Texto, conteniendo:

- Título y subtítulo (presentar versiones en español, portugués e inglés)

- Resumen: en español o portugués, no debe exceder 250 palabras. Debe incluir las siguientes secciones: objetivos, material y métodos, resultados y conclusiones. No usar abreviaciones o siglas.

- Palabras clave: Al final del resumo deben ser incluidas a lo sumo seis (6) palabras llaves, en consonancia con los "Descritores para Ciências da Saúde" - BIREME (DeCS). Consulta electrónica por la dirección http: / / decs.bvs.br/

- Abstract: Versión en inglés del resumen.

- Keywords: Palabras claves en su versión en inglés.

- Introducción: Presentando el estado actual del conocimiento con relación al tema, indicando las hipótesis y objetivos del trabajo.

- Material y Métodos: Debe ser presentado con detalles suficientes para ir a permitir la confirmación de las observaciones. Especificar la población del estudio (con el tipo de muestra y la técnica ). Citar los métodos estadísticos utilizados y los programas de ordenador empleados. Presentar evidencias claras de que los principios éticos fueron seguidos. 
- Resultados: Debe describir los resultados obtenidos, considerándose los objetivos propuestos. No repetir los datos de tablas o gráficos.

- Tablas: deberán ser numeradas consecutivamente en números arábicos según la orden que aparecen en el texto, estar en páginas separadas y presentar una legenda en la parte superior. Las notas de rodapié deberán ser indicadas por asteriscos y restrictas al mínimo indispensable.

- Fotografías/figuras: Deberá ser enviada en archivo JPG o TIF con resolución mínima de 300DPI, acompañada con legenda. Los editores reservan el derecho de publicarlas en colores o negro y blanco. Las fotos de observaciones microscópicas deberán poseer la indicación de la escala/ampliación efectuada. Si la figura ya fue publicada se debe mencionar el autor y presentar la autorización.

- Discusión: Presentar como una sección independiente de los resultados. Considerar principalmente los aspectos innovadores e importantes del estudio y relatar las observaciones relevantes de otros estudios. Mencionar los beneficios y limitaciones del trabajo.

- Conclusiones: Debe resumir los principales hallazgos, sugerencias o recomendaciones.

- Abreviaturas y símbolos: Todas las abreviaciones deben tener su descripción por extenso, entre paréntesis, en la primera vez en que son mencionadas. No utilizarlas en el título, resumo o conclusiones.

- Agradecimientos: cuando considerado necesario y en relación las personas o instituciones

- Referencias: Deberá contener solamente las citadas en el texto y estar numeradas (números arábicos) en consonancia con la orden de aparición en el texto, en estilo Vancouver en consonancia con los ejemplos a continuación. Adopta las normas de publicación del International Committee of Medical Journal Editors, disponible en la dirección electrónica HTTP:/ / www.nlm.nih.gov/bsd/uniform_requirements.html. Debe utilizarse solamente las referencias esenciales al desarrollo del artículo y no exceder 30 referencias; para trabajos de revisión ese número deberá ser a lo sumo 50.

\section{Ejemplos:}

\section{Artículo de revista}

Mount GJ. Clinical requirements for a successful "sandwich"-dentine to glass ionomer cement to composite resin. Aust Dent J 1989;34:259-65.

Ferrari M. Use of glass ionomers as bondings, linings, or bases. In: Davidson CL, Mjor IA, eds. Advances in Glass Ionomer Cements. Berlin, Germany / Chicago, Ill: Quintessence Publishing Co; 1999:137-48.

Croll TP, Bar-Zion Y, Segura A, et al. Clinical performance of resin-modified glass ionomer cement restoration in primary teeth: A retrospective evaluation. J Am Dent Assoc 2001;132:1110-6.

American Academy of Pediatric Dentistry. Oral Health Policy on Interim Therapeutic Restoration. Reference Manual 2008-09. Pediatr Dent 2009;30:38.

\section{Libro}

Pinkhan JR, Casamassimo PS, Fields HW, McTigue, DLNowak A.Pediatric Dentistry: Infancy Through Adolescence. $4^{\text {a }}$ ed.Philadelphia, Pa:WBSaunders; 2005. 


\section{Capítulo de libro}

PS Casamassimo Childrens Pulpa Dentaria capítulo 3 en: A.Pediatric Dentistry:Infancy Through Adolescence. $4^{\mathrm{a}}$ ed.Philadelphia,Pa:WBSaunders; 2005.

\section{Referencia electrónica}

Morse SS. Factors in the emergence of infectious diseases.Emerg Infect Dis Accesado (2005 Jun 5). Disponible en: URL:http:/ / www.cdc.gov/ncidod/EID/ eid.htm

\section{Envío de trabajos}

- Por correo electrónico (e-mail)

Para: editor.alop-odontopediatria.org,guidoperona54@hotmail.com, fabianfraiz@gmail.com

Asunto: Publicación Artículo Revista de Odontopediatría Latinoamericana.

Cuerpo: Título de artículo, nombre de autor, solicitando revisión y publicación.

Archivo adjunto: Artículo en Word, archivos de figuras, tablas. 